\title{
Untangling EMT's functions
}

Although epithelial-mesenchymal transition (EMT) is commonly believed to contribute to metastasis, definitive in vivo evidence to support this theory is lacking. Two studies in Nature report that EMT is not required for metastasis in mouse tumour models; however, EMT can contribute to resistance to chemotherapy.

Fischer et al. generated mouse models of breast cancer that undergo spontaneous metastasis, and in which EMT can be traced. Mice expressing either polyomavirus middle T antigen (PyMT) or Neu (also known as Erbb2) in the mammary gland were engineered to also express a Cre-switchable fluorescent marker such that cells expressing fibroblast-specific protein 1 (Fsp1; which is activated early during EMT) would switch from expressing red fluorescent protein (RFP) to express green fluorescent protein (GFP). Therefore, cells that had undergone EMT were labelled with GFP (this was confirmed in vitro); importantly, this GFP expression is irreversible, so any cells subsequently undergoing mesenchymal-epithelial transition $(\mathrm{MET})$ remain $\mathrm{GFP}^{+}$. The development of primary tumours and spontaneous lung metastases in these tri-PyMT and tri-Neu mouse models was indistinguishable from controls. Unexpectedly, the tumour cells in the metastatic lesions in these mice were $\mathrm{RFP}^{+}$, indicating that they had never expressed Fsp1.

As EMT could potentially occur without Fsp1 expression, the authors also generated tri-PyMT mice that switched from $\mathrm{RFP}^{+}$to $\mathrm{GFP}^{+}$when vimentin (Vim, another common EMT marker) was expressed. Metastases in these tri-PyMT/Vim mice were also $\mathrm{RFP}^{+}$. These data were confirmed using orthotopic injection of tri- $P y M T$ or tri-PyMT/Vim cells into wild-type mice to ensure that only tumour cells that had undergone EMT would be $\mathrm{GFP}^{+}$. As it remained possible that some cells might undergo EMT without expressing either Fsp1 or Vim, the authors also inhibited EMT in tri-PyMT cells by expressing the microRNA miR-200, which targets the EMT transcription factors Zeb1 and Zeb2. Although EMT was blocked in these cells, metastasis was not inhibited following orthotopic injection.

EMT also has a reported role in resistance to chemotherapy. Treatment of mice bearing orthotopic tri-PyMT tumours with cyclophosphamide reduced primary tumour size. In contrast to untreated mice, many metastatic lesions in the treated mice contained a substantial number of $\mathrm{GFP}^{+}$cells, and several lines of evidence showed these metastatic cells to be more resistant to cyclophosphamide in a manner that depended on induction of EMT. These $\mathrm{GFP}^{+}$cells expressed many factors implicated in proliferation and chemotherapy resistance, although the specific mechanisms underlying resistance were not determined.

Zheng, Carstens et al. reported similar data in the KPC mouse model of pancreatic ductal adenocarcinoma (PDAC), in which mice develop metastatic tumours owing to expression of mutant p53 and KRAS-G12D in pancreatic cells. KPC mice were crossed to mice lacking one of two EMT transcription factors: SNAI1 or TWIST1. Although EMT was suppressed in these mice, they had a similar tumour burden and no change in overall survival compared with KPC mice.
Furthermore, EMT suppression did not affect the number of circulating tumour cells, the ability of tumour cells to form tumour spheres in vitro or colonize the lung following intravenous injection, or the overall frequency of metastasis. These authors also found that suppression of EMT in KPC mice reduced PDAC progression and increased survival following treatment with the nucleoside analogue gemcitabine, an effect shown to correlate with the upregulation of nucleoside transporters. KTC mice, which express mutant KRAS and lack transforming growth factor- $\beta$ receptor 2 (TGF $\beta$ R2), also develop metastatic PDAC, and as in KPC mice, knockout of Snail did not prevent metastasis but did enhance sensitivity to gemcitabine.

These two studies provide intriguing evidence that although therapeutic inhibition of EMT might not prevent metastasis, combining chemotherapy with EMT inhibition might help to prevent the emergence of resistance.

Sarah Seton-Rogers

ORIGINAL ARTICLES Fischer, K. R. et al. Epithelial-to-mesenchymal transition is not required for lung metastasis but contributes to chemoresistance. Nature http://dx.doi. org/10.1038/nature15748 (2015)|Zheng, X., Carstens, J. L. et al. Epithelial-to-mesenchymal transition is dispensable for metastasis but induces chemoresistance in pancreatic cancer. Nature http://dx.doi.org/10.1038/nature16064 (2015) 